\title{
Effect of Integrated Nutrient Management on Yield and Quality of Basmati Rice Varieties
}

\author{
Uppu Sai Sravan ${ }^{1,2} \&$ Shiv Prakash Singh ${ }^{1}$ \\ ${ }^{1}$ Department of Agronomy, Institute of Agricultural Sciences, Banaras Hindu University, Varanasi, Uttar \\ Pradesh, India \\ ${ }^{2}$ ICAR-CRIDA, Hyderabad, Telangana, India
}

Correspondence: Uppu Sai Sravan, ICAR-CRIDA, Santoshnagar, Hyderabad, Telangana, India. Tel: 91-0898-5020-898. E-mail: saisravan050@gmail.com

Received: January 7, 2019 Accepted: February 14, $2019 \quad$ Online Published: April 15, 2019

doi:10.5539/jas.v11n5p93 URL: https://doi.org/10.5539/jas.v11n5p93

\begin{abstract}
A 2-year study conducted to determine the effect of integrated nutrient management on yield and quality of basmati rice varieties in non-traditional area with 54 plots in split plot design. Variety HUBR 10-9 produced $18.8 \%$ higher mean grain yield and superior quality parameters than HUBR 2-1. Mean milling, head rice recovery, amylose content and alkali digestion value noted higher with HUBR $10-9$ by $4.1 \%, 4.1 \%, 8.5 \%$ and $15.1 \%$ over HUBR $2-1$, respectively. Addition of $75 \%$ recommended dose of fertilizers with $25 \%$ recommended dose of nitrogen as farmyard manure produced higher mean values by $3.1 \%, 4.2 \%$ and $4.0 \%$ for hulling, milling and head rice recovery respectively over $100 \%$ recommended dose applied as inorganic sources. Combined use of bio-inoculants (blue green algae plus Azospirillum) exhibited higher values for yield and quality parameters. HUBR $10-9$ be grown using $75 \%$ recommended dose of fertilizers with $25 \%$ nitrogen as farmyard manure and blue green algae plus Azospirillum for enhancement in yield and quality in non-traditional areas of eastern Uttar Pradesh. Further investigation required under diverse conditions.
\end{abstract}

Keywords: basmati rice, bio-inoculants, nitrogen management, quality parameters

\section{Introduction}

Rice (Oryza sativa L.) is the second most important food crop for more than half of the world population, provides carbohydrates, proteins, vitamins and is an important source of nutrients due to its every day consumption (Heinemann, Fagundes, Pinto, Penteado, \& Lanfer-Marquez, 2005). Basmati is a globally reputed aromatic group of rice, having pleasant aroma, superfine grain with extensive kernel elongation and soft texture of cooked rice (Juliano, 1972). Basmati rice cultivation is traditionally practiced on north and north-western regions of the Indian sub-continent with best quality rice's produced on both sides of Indus River. Popular basmati rice varieties traditionally grown namely Basmati 370, Basmati 386 and Taraori Basmati are low yielder, tall in stature and lodge at higher levels of inorganic fertilizers. Growing of these cultivars in non-traditional areas causes reduction in grain size and aroma with enhanced breakage. Recently released Basmati varieties namely HUBR 2-1 (non-traditional areas) and HUBR 10-9 (basmati geographical indication areas) are recommended for cultivation in India can address poor yield and quality associated problems. High yield potential and quality advantage of these varieties over traditional one may open new avenues for the export of high quality rice (Singh, Jaiswal, \& Madhavilatha, 2006). Climatic limitations restrict cultivation of basmati rice in non-traditional areas because of inferior quality and are a major constraint. Temperature plays vital role on grain quality in non-traditional areas. From flowering to maturity, temperature range of $25{ }^{\circ} \mathrm{C}$ to $32{ }^{\circ} \mathrm{C}$ with relatively cooler nights $\left(20-25^{\circ} \mathrm{C}\right)$ and moderate humidity are indispensable for the development of proper grain and aroma. Higher temperature during grain filling affects the yield and amylose content of basmati rice (Ahmed et al., 2015). Physico-chemical and cooking characteristics of rice varieties show significant variations, mainly due to their genetic constitution. Genotypic factor is considered as most prominent which determines the plant response to different soil conditions and other external factors (Chandel et al., 2010).

Nutrient management in rice is of pivotal importance to achieve sustainability in production. Imbalanced fertilizer use results stagnant yields and deteriorated soil health (Surekha, Rao, Shobha Rani, Latha, \& Kumar, 2013). Unavailability of chemical fertilizers and their escalating cost creating problem. Hence, reduction in the 
dose of chemical fertilizers and enhanced use of organic inputs like farmyard manure and biofertilizers may improve soil health, productivity and profitability of rice (Singh, Kumar, \& Pal, 2008). Biofertilizers and organic sources are cheap, eco-friendly and supply nutrients (both macro and micro) to the crop for prolonged period. Farmyard manure acts as a soil conditioner improves physical, chemical and biological properties of the soil and provides congenial conditions for the growth of microbial populations. Hence, profuse root growth and greater nutrient accumulation results in better yield and grain quality (Adhikari, Mishra, \& Mishra, 2005). Nitrogen is most important nutrient; varying organic and inorganic nitrogen sources have significant influence and crucial role on grain quality. Integrated nutrient management including biofertilizers, organic and chemical sources may improve productivity and the grain quality. Biofertilizers alone or in combinations with organic manures improve the quality parameters of scented rice (Dixit \& Gupta, 2000; Quyen \& Sharma, 2003). Head rice recovery and alkali value of scented rice varieties improved due to application of farmyard manure (Pandey, Sarawgi, Rastogi, \& Tripathi, 1999). Lower doses of organic manures improved the quality parameters like hulling, milling and head rice recovery than higher doses (Saha et al., 2007). Milling, head rice recovery, kernel length and length: breadth ratio after cooking of rice significantly improved when organic manures applied in combination with inorganic (Pandey et al., 1999; Quyen \& Sharma, 2003; Srivastava, Srivastava, Singh, \& Shrivastava, 2009). Blue green algae (BGA) and Azospirillum may be utilized successfully in wetland rice to supplement part of nitrogen requirement with ecological and economic significance.

Keeping in view the above facts an investigation was conducted in non-traditional area of north eastern plains of eastern Uttar Pradesh. We assessed the response of basmati varieties to integrated nutrient management to ascertain equivalent productivity as with sole use of inorganic sources. Therefore, the objective of present study was to determine the effects of integrated nutrient management on yield potential and quality of the basmati varieties in non-traditional areas.

\section{Material and Methods}

\subsection{Experimental Site}

A field investigation was carried out during rainy seasons of 2014 and 2015 at Agricultural Research Farm, Banaras Hindu University, Varanasi $\left(25^{\circ} 32^{\prime} \mathrm{N}, 82^{\circ} 97^{\prime} \mathrm{E}\right.$, a sub-tropical semi-arid climate, at an elevation of $86 \mathrm{~m}$ above the mean sea level). Rainfall was $764.6 \mathrm{~mm}$ and $871.5 \mathrm{~mm}$ in 2014 and 2015, respectively annually coupled with $29.1{ }^{\circ} \mathrm{C}$ and $28.6{ }^{\circ} \mathrm{C}$ during the cropping. Soil of the experimental site was Cambisols with pH of 7.33 , EC of 0.40 , soil organic carbon of $0.44 \%, 225.79 \mathrm{~kg} \mathrm{ha}^{-1}$ available nitrogen (N), $20.96 \mathrm{~kg} \mathrm{ha}^{-1}$ available phosphorus (P) and $180.77 \mathrm{~kg} \mathrm{ha}^{-1}$ available potassium (K). Prior to experimental crops, composite soil samples were drawn randomly $(0-30 \mathrm{~cm})$ from 10 places, dried and passed through $2.0 \mathrm{~mm}$ sieve and subjected to chemical analyses.

\subsection{Experimental Description}

Experiment was laid out in split plot design replicated thrice; comprising of two varieties: HUBR 2-1 $\left(\mathrm{V}_{1}\right)$; HUBR 10-9 $\left(\mathrm{V}_{2}\right)$ and three fertility levels: $100 \%$ recommended dose of fertilizer (RDF) $\left[\mathrm{N}_{1}\right] ; 75 \% \mathrm{RDF}+25 \%$ recommended dose of nitrogen $(\mathrm{RDN})$ as $\mathrm{FYM}\left[\mathrm{N}_{2}\right] ; 50 \% \mathrm{RDF}+50 \% \mathrm{RDN}$ as $\mathrm{FYM}\left[\mathrm{N}_{3}\right]$ in main plots and three bio-inoculants: BGA $\left(\mathrm{B}_{1}\right)$; Azospirillum $\left(\mathrm{B}_{2}\right)$; $\mathrm{BGA}+$ Azospirillum $\left(\mathrm{B}_{3}\right)$ in sub plots. The experimental area was ploughed twice with tractor drawn cultivator, planked and puddle twice with rotovator in about $10 \mathrm{~cm}$ standing water. Thereafter, $4.2 \mathrm{~m}$ long and $4.0 \mathrm{~m}$ wide plots were laid out followed by transplanting of seedlings as per plan. RDF used was $120 \mathrm{~kg} \mathrm{~N} \mathrm{ha}^{-1}, 60 \mathrm{~kg} \mathrm{P}_{2} \mathrm{O}_{5} \mathrm{ha}^{-1}$ and $60 \mathrm{~kg} \mathrm{~K}_{2} \mathrm{O} \mathrm{ha}^{-1}$. FYM $(0.51 \% \mathrm{~N})$ was used to supply $25 \%\left(6 \mathrm{tha}^{-1}\right)$ and $50 \%\left(12 \mathrm{tha}^{-1}\right)$ of the RDN applied three days before transplanting. Blue green algae applied at the rate of $10 \mathrm{~kg} \mathrm{ha}^{-1}$ on $10^{\text {th }}$ day after transplanting. Azospirillum brasilense mother culture was diluted 10 times and kept in the growth chamber for 3-4 days. Inoculants suspension was prepared using water in the ratio of 1:10; roots of rice seedlings were dipped in liquid Azospirillum for 30 minutes and transplanted immediately in respective plots. The recommended dose of $\mathrm{N}$ was supplied in form of inorganic fertilizer and/or FYM. Half of the $\mathrm{N}$ and total $\mathrm{P}$ and $\mathrm{K}$ were applied at transplanting and remaining half $\mathrm{N}$ was top dressed in two equal splits at active tillering and panicle initiation stages. All the treatments were applied to their respective plots as per scheduled plan. Sources of fertilizers were urea $(46 \% \mathrm{~N})$, diammonium phosphate $(18 \% \mathrm{~N}, 46 \%$ $\left.\mathrm{P}_{2} \mathrm{O}_{5}\right)$ and muriate of potash $\left(60 \% \mathrm{~K}_{2} \mathrm{O}\right)$. A seed rate of $30 \mathrm{~kg} \mathrm{ha}^{-1}$ was adopted to raise nursery on $20^{\text {th }}$ and $22^{\text {nd }}$ June during 2014 and 2015, respectively. Two seedlings per hill transplanted at spacing of $20 \times 15 \mathrm{~cm}$ on $19^{\text {th }}$ July and $21^{\text {st }}$ July during first and second year, respectively. HUBR 2-1 was harvested at 130 days after sowing while HUBR 10-9 a week later (137 days) from an area of $10.56 \mathrm{~m}^{2}$. 


\subsection{Yield}

Produce of each net plot was threshed separately and grains thus obtained were winnowed, cleaned and weighed. Harvested grains contained 14 per cent moisture measured by moisture meter. Straw yield measured by subtracting grain yield from total biological yield and expressed in $\mathrm{q} \mathrm{ha}^{-1}$.

\subsection{Grain Quality Evaluation}

To assess quality characters, $100 \mathrm{~g}$ sample of rough rice (paddy) from each plot used. Rough rice samples were dehusked to produce brown rice with Satake THU-35A laboratory rice sheller (comprises of rubber roll, rotates at $1900 \mathrm{rpm}$ and an identical rubber roll which rotates at $1000 \mathrm{rpm}$ in a counter rotation). As the kernels pass through the gap between the rolls, the rubber rolls impart a shearing and frictional force to kernels; hulls were dislodged by suction fan (Siebenmorgen, Jia, Qin, \& Schluterman, 2006). Brown rice was milled in Kett T-2 rice polisher for 30 seconds to obtain milled rice and expressed as hulling and milling percentage (Khush, Paule, \& Dela Cruz, 1979). Kernels with length greater than or equal to three-fourth of its total length were considered as head rice and expressed as head rice recovery (Zhou et al., 2015). Ten head rice grains from each plot were lined up length and breadth wise on graph paper to measure the kernel length and breadth before cooking. Afterwards these rice kernels were pre-soaked for 15 minutes in $20 \mathrm{ml}$ distilled water and heated to $98^{\circ} \mathrm{C}$ for 10 minutes. Thereafter, length and breadth of cooked kernels were measured on graph paper. Elongation ratio was computed by dividing the cumulative length of 10 cooked kernels by respective length of raw kernels (Juliano \& Perez, 1984).

N content was estimated by Kjeldahl procedure (Jackson, 1973) and grain protein content was calculated by multiplying $\mathrm{N}$ content with 6.25 (AOAC, 1990). Protein yield was determined by multiplying the protein content with their respective yield. Twenty whole grain (milled rice) samples were oven dried at $60{ }^{\circ} \mathrm{C}$ and ground in a cyclone Mill and starch solution is prepared. Amylose content was determined using the simplified automated iodine-colorimetric procedure and absorbance of the starch solution was measured at $620 \mathrm{mu}$ with spectrophotometer (Juliano, 1971). Alkali digestion test was performed and starchy endosperm is rated visually based on 7-point numerical spreading scale (Dela Cruz \& Khush, 2000).

\subsection{Statistical Analysis}

Analysis of variance (ANOVA) was performed as described for the split plot design (Gomez \& Gomez, 1984) using statistical package SPSS software (version 23.0; IBM Corp.; Armonk, NY, USA), and means between treatments were compared with the least significant difference $(L S D)$ test $(p<0.05)$. All graphs were prepared in excel and mean values are reported with standard error of means.

\section{Results and Discussion}

\subsection{Yield}

Yield of basmati rice varieties varied significantly with fertility levels and bio-inoculants during both the years (Table 1). Variety HUBR 10-9 registered $18.8 \%$ higher mean grain yield than HUBR 2-1. Yield (grain and straw) enhancement with HUBR 10-9 was due to improvement in growth parameters, yield components, translocation of photosynthates from source to sink and genetic makeup of genotype. Variations in yields of cultivars were also reported (Gautam, Kumar, Shivay, \& Mishra, 2008). Improved yields with integrated nutrient management $\left(\mathrm{N}_{2}\right)$ were due to instantaneous and rapid supply of nutrients through chemical fertilizers and steady supply through mineralization of FYM for prolonged period. Organics along with chemical fertilizers perhaps minimised the $\mathrm{N}$ loss and increased availability throughout the crop growth period through formation of organic-mineral complexes (Yaduvanshi, 2003). Similar results on rice yields were reported due to integrated application of chemical fertilizer and organic manures (Singh, Pandey, Nanda, \& Gupta, 2018; Tang et al., 2018). Bio-inoculants followed the order of $B_{3}, B_{1}$ and $B_{2}$ (Table 1). However, combined application $\left(B_{3}\right)$ increased the mean grain yield by $4.0 \%$ and $7.2 \%$ than $\mathrm{B}_{1}$ and $\mathrm{B}_{2}$, respectively. Increment in yield by combined application of bio-inoculants could be attributed to their synergistic effect (Nanda, Sravan, A. Singh, \& S. P. Singh, 2016). BGA facilitated efficient nitrogen utilization and released synthesised nitrogenous compounds either by decomposition of the cells or by excretion (Nayak, Prasanna, Pabby, Dominic, \& Singh, 2004). Release of growth promoting substances from Azospirillum enhanced the root growth and its surface area and improved accessibility for mineral absorption (Murthy \& Ladha, 1988). 
Table 1. Effect of varieties, fertility levels and bio-inoculants on grain yield, straw yield, hulling, milling and head rice recovery of rice

\begin{tabular}{|c|c|c|c|c|c|c|}
\hline & Treatments & Grain yield $\left(\mathrm{q} \mathrm{ha}^{-1}\right)$ & Straw yield $\left(\mathrm{q} \mathrm{ha}{ }^{-1}\right)$ & Hulling (\%) & Milling (\%) & Head rice recovery $(\%)$ \\
\hline \multirow[t]{11}{*}{2014} & Varieties & & & & & \\
\hline & $\mathrm{V}_{1}$ & $47.82 \pm 0.85^{\mathrm{b}} *$ & $72.74 \pm 0.95^{\mathrm{b}}$ & $70.70 \pm 0.45^{\mathrm{b}}$ & $61.48 \pm 0.58^{\mathrm{b}}$ & $52.28 \pm 0.43^{\mathrm{b}}$ \\
\hline & $\mathrm{V}_{2}$ & $57.16 \pm 0.85^{\mathrm{a}}$ & $82.02 \pm 0.95^{\mathrm{a}}$ & $73.42 \pm 0.45^{\mathrm{a}}$ & $64.04 \pm 0.58^{\mathrm{a}}$ & $54.34 \pm 0.43^{\mathrm{a}}$ \\
\hline & Fertility levels & & & & & \\
\hline & $\mathrm{N}_{1}$ & $52.39 \pm 1.04^{b}$ & $76.44 \pm 1.16^{\mathrm{ab}}$ & $71.09 \pm 0.56^{\mathrm{b}}$ & $61.31 \pm 0.71^{\mathrm{b}}$ & $52.07 \pm 0.52^{\mathrm{b}}$ \\
\hline & $\mathrm{N}_{2}$ & $56.17 \pm 1.04^{\mathrm{a}}$ & $80.08 \pm 1.16^{\mathrm{a}}$ & $73.37 \pm 0.56^{\mathrm{a}}$ & $64.20 \pm 0.71^{\mathrm{a}}$ & $54.26 \pm 0.52^{\mathrm{a}}$ \\
\hline & $\mathrm{N}_{3}$ & $48.92 \pm 1.04^{\mathrm{c}}$ & $75.63 \pm 1.16^{\mathrm{b}}$ & $71.71 \pm 0.56^{\mathrm{ab}}$ & $62.75 \pm 0.71^{\mathrm{ab}}$ & $53.59 \pm 0.52^{\mathrm{ab}}$ \\
\hline & Bio-inoculants & & & & & \\
\hline & $\mathrm{B}_{1}$ & $52.13 \pm 0.83^{\mathrm{ab}}$ & $77.71 \pm 0.94^{\mathrm{ab}}$ & $71.69 \pm 0.47^{\mathrm{ab}}$ & $62.60 \pm 0.46^{\mathrm{ab}}$ & $53.19 \pm 0.45^{\mathrm{ab}}$ \\
\hline & $\mathrm{B}_{2}$ & $51.06 \pm 0.83^{\mathrm{b}}$ & $75.11 \pm 0.94^{\mathrm{b}}$ & $71.43 \pm 0.47^{\mathrm{b}}$ & $61.98 \pm 0.46^{\mathrm{b}}$ & $52.54 \pm 0.45^{\mathrm{b}}$ \\
\hline & $\mathrm{B}_{3}$ & $54.29 \pm 0.83^{\mathrm{a}}$ & $79.32 \pm 0.94^{\mathrm{a}}$ & $73.07 \pm 0.47^{\mathrm{a}}$ & $63.69 \pm 0.46^{\mathrm{a}}$ & $54.20 \pm 0.45^{\mathrm{a}}$ \\
\hline \multirow[t]{11}{*}{2015} & Varieties & & & & & \\
\hline & $\mathrm{V}_{1}$ & $50.39 \pm 1.06^{\mathrm{b}}$ & $77.10 \pm 1.15^{\mathrm{b}}$ & $72.11 \pm 0.36^{\mathrm{b}}$ & $63.44 \pm 0.43^{\mathrm{b}}$ & $53.61 \pm 0.39^{\mathrm{b}}$ \\
\hline & $\mathrm{V}_{2}$ & $59.55 \pm 1.06^{\mathrm{a}}$ & $86.48 \pm 1.15^{\mathrm{a}}$ & $75.04 \pm 0.36^{\mathrm{a}}$ & $65.96 \pm 0.43^{\mathrm{a}}$ & $55.88 \pm 0.39^{\mathrm{a}}$ \\
\hline & Fertility levels & & & & & \\
\hline & $\mathrm{N}_{1}$ & $54.97 \pm 1.30^{\mathrm{b}}$ & $81.61 \pm 1.41^{\mathrm{ab}}$ & $72.54 \pm 0.44^{\mathrm{b}}$ & $63.58 \pm 0.53^{\mathrm{b}}$ & $53.84 \pm 0.48^{\mathrm{b}}$ \\
\hline & $\mathrm{N}_{2}$ & $59.18 \pm 1.30^{\mathrm{a}}$ & $84.75 \pm 1.41^{\mathrm{a}}$ & $74.73 \pm 0.44^{\mathrm{a}}$ & $65.85 \pm 0.53^{\mathrm{a}}$ & $55.87 \pm 0.48^{\mathrm{a}}$ \\
\hline & $\mathrm{N}_{3}$ & $50.76 \pm 1.30^{\mathrm{c}}$ & $79.00 \pm 1.41^{\mathrm{b}}$ & $73.45 \pm 0.44^{b}$ & $64.66 \pm 0.53^{\mathrm{ab}}$ & $54.51 \pm 0.48^{\mathrm{b}}$ \\
\hline & Bio-inoculants & & & & & \\
\hline & $\mathrm{B}_{1}$ & $54.92 \pm 1.06^{\mathrm{ab}}$ & $81.95 \pm 1.08^{\mathrm{ab}}$ & $73.51 \pm 0.29^{\mathrm{ab}}$ & $64.58 \pm 0.37^{\mathrm{ab}}$ & $54.65 \pm 0.35^{\mathrm{ab}}$ \\
\hline & $\mathrm{B}_{2}$ & $52.87 \pm 1.06^{\mathrm{b}}$ & $79.20 \pm 1.08^{\mathrm{b}}$ & $73.03 \pm 0.29^{b}$ & $63.98 \pm 0.37^{\mathrm{b}}$ & $54.09 \pm 0.35^{\mathrm{b}}$ \\
\hline & $\mathrm{B}_{3}$ & $57.13 \pm 1.06^{\mathrm{a}}$ & $84.21 \pm 1.08^{\mathrm{a}}$ & $74.18 \pm 0.29^{\mathrm{a}}$ & $65.53 \pm 0.37^{\mathrm{a}}$ & $55.49 \pm 0.35^{\mathrm{a}}$ \\
\hline
\end{tabular}

Note. *Mean values \pm SE. Mean values with different superscript letters denote significant difference $(\mathrm{p}<0.05)$. $\mathrm{V}_{1}=$ HUBR 2-1; $\mathrm{V}_{2}=$ HUBR $10-9 ; \mathrm{N}_{1}=100 \% \mathrm{RDF} ; \mathrm{N}_{2}=75 \% \mathrm{RDF}+25 \% \mathrm{RDN}$ as FYM; $\mathrm{N}_{3}=50 \% \mathrm{RDF}+$ $50 \% \mathrm{RDN}$ as FYM; $\mathrm{B}_{1}=\mathrm{BGA} ; \mathrm{B}_{2}=$ Azospirillum $; \mathrm{B}_{3}=\mathrm{BGA}+$ Azospirillum.

\subsection{Hulling, Milling and Head Rice Recovery}

Grain quality traits are economically important particularly for basmati genotypes. The issue of grain quality inheritance is more complex and the quality traits are governed by several (major and minor) quantitative trait loci (QTLs). Hulling, milling and head rice recovery of varieties distinctly varied with fertility levels and bio-inoculants in both years (Table 1). HUBR 10-9 produced significantly greater hulling, milling and head rice recovery compared to HUBR 2-1 during 2014 and 2015, respectively. Improvement in milled rice noted to the extent of $4.1 \%$ by HUBR $10-9$ over HUBR 2-1. Variation in quality parameters depends on the grain type, variety, cultural practices and temperature during grain filling to maturity stage and drying condition (Dela Cruz, Kumar, Kaushik, \& Khush, 1989; Binodh, Kalaiyarasi, Thiyagarajan, \& Manonmani, 2006; Gautam et al., 2008). Head rice recovery is a genetic trait but environmental factors and post harvest handling play vital role and persuade the grain breakage during milling (Fan, Siebenmorgen, \& Yang, 2000). Similar findings on quality parameters were reported (Ghosh, Mandal, Mandal, Lodh, \& Dash, 2004).

Integrated nutrient management $\left(\mathrm{N}_{2}\right)$ recorded maximum values for quality characters (Table 1). Application of entire nutrients as inorganic source $\left(\mathrm{N}_{1}\right)$ exhibited $4.2 \%$ lower milling than $\mathrm{N}_{2}$. FYM supplied macro and micro nutrients in optimum quantities, resulted enhanced quality. Quality parameters improved with combined use of organic and inorganic nutrient sources (Dixit \& Gupta, 2000), incorporation of FYM significantly increased the hulling per cent in rice (Prakash, Bhadoria, \& Rakshit, 2002). Maximum improvement in hulling, milling and head rice recovery occurred by BGA + Azospirillum $\left(\mathrm{B}_{3}\right)$ was due to enhanced availability of nitrogen for longer duration. Bio-inoculants when interacted with organic and inorganic fertilizer sources supplied nitrogen consistently and showed marked improvement. Differences in hulling, milling and head rice recovery with bio-inoculants were also reported (Quyen \& Sharma, 2003; Davari \& Sharma, 2010).

The interaction effect between varieties and fertility levels $(\mathrm{V} \times \mathrm{N})$ was found significant during both the years for hulling (Figures 1A and 1B), milling (Figures 1C and 1D) and head rice recovery (Figures 1E and 1F). Optimum nutrient supply with integrated nutrient management treatments $\left(\mathrm{N}_{2}\right.$ and $\left.\mathrm{N}_{3}\right)$ result improved quality parameters by HUBR 10-9 than HUBR 2-1 due to genetic constitution and exploitation of its higher yield 
potential. Longer sunshine hours during grain filling resulted better translocation of photosynthates to sink thus improved the quality. The results are in accordance with Perez, Juliano, Liboon, Alcantara, \& Cassman (1996). Interaction effect found significant between varieties and bio-inoculants for hulling during both the years (Figures 2A and 2B). Alone use of BGA or combined application (BGA and Azospirillum) improved hulling of HUBR 10-9. Production of higher algal biomass and availability of nitrogen from its decomposition might have resulted in better quality. Fertility levels and bio-inoculants interacted significantly for hulling during first year (Figure 3A). Integration of $75 \%$ RDF plus $25 \%$ RDN as FYM with bio-inoculants enhanced hulling since combined use of inorganic, organic and bio-inoculants maintained nutrients supply throughout crop growth period resulted better quality. Similar results reported by other workers (Davari \& Sharma, 2010; Hargilas \& Sharma, 2015).

(A)

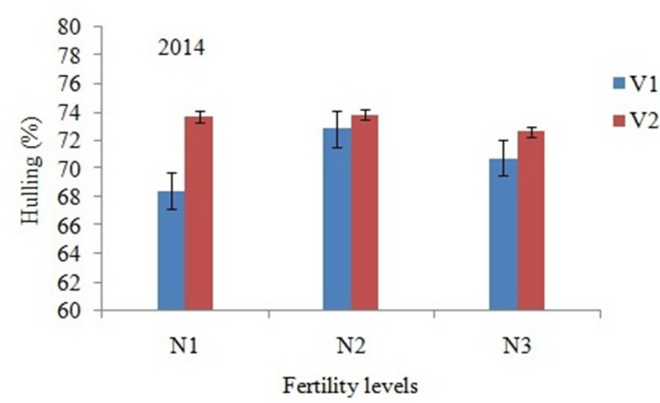

(C)

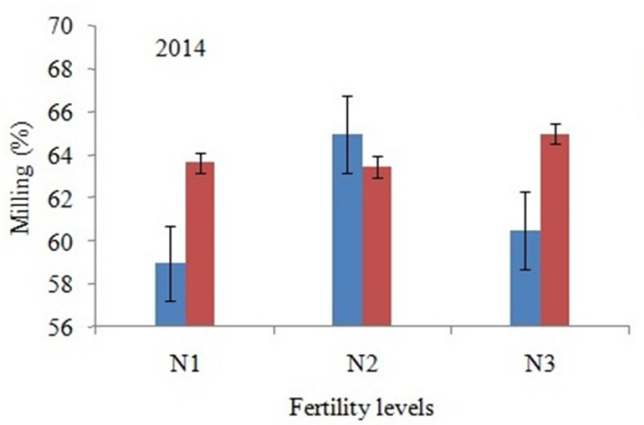

(E)

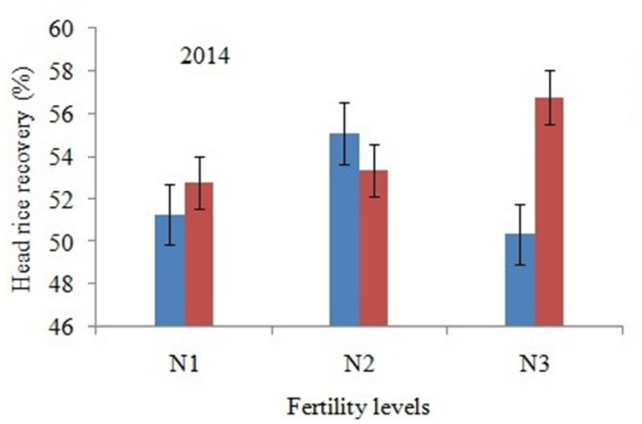

(B)

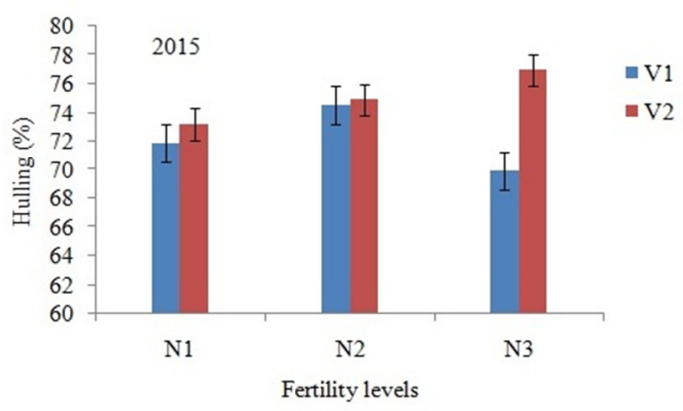

(D)

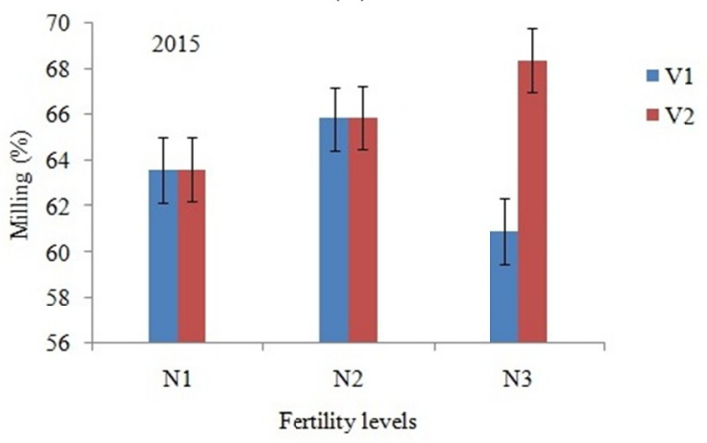

(F)

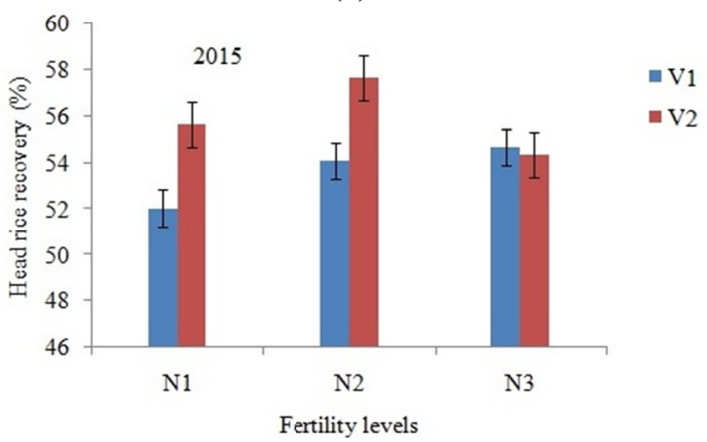

Figure 1. Interaction of varieties and fertility levels $(\mathrm{V} \times \mathrm{N})$ for $(\mathrm{A})$ hulling during $2014(\mathrm{p}<0.05)$; (B) hulling during 2015 (p < 0.01); (C) milling during 2014 (p<0.05); (D) milling during 2015 ( $p<0.01)$; (E) head rice recovery during $2014(\mathrm{p}<0.01)$; $(\mathrm{F})$ head rice recovery during $2015(\mathrm{p}<0.05)$ of rice; $\mathrm{V}_{1}=\mathrm{HUBR}_{2}-1 ; \mathrm{V}_{2}=$ HUBR 10-9; $\mathrm{N}_{1}=100 \% \mathrm{RDF} ; \mathrm{N}_{2}=75 \% \mathrm{RDF}+25 \% \mathrm{RDN}$ as FYM; $\mathrm{N}_{3}=50 \% \mathrm{RDF}+50 \% \mathrm{RDN}$ as FYM; vertical bars represent \pm standard error of the mean 
(A)

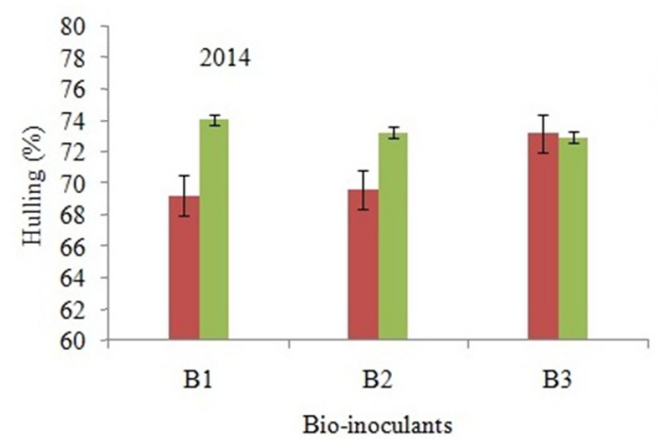

(B)

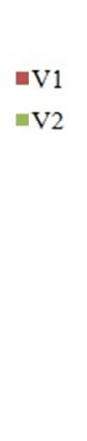

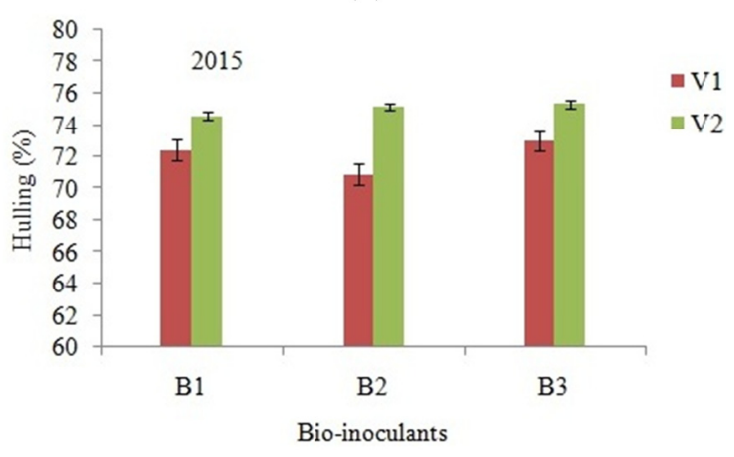

Figure 2. Interaction of varieties and bio-inoculants $(\mathrm{V} \times \mathrm{B})$ for $(\mathrm{A})$ hulling during $2014(\mathrm{p}<0.01)$; (B) hulling during $2015(\mathrm{p}<0.05)$ of rice; $\mathrm{V}_{1}=$ HUBR $2-1 ; \mathrm{V}_{2}=$ HUBR $10-9 ; \mathrm{N}_{1}=100 \% \mathrm{RDF} ; \mathrm{N}_{2}=75 \% \mathrm{RDF}+25 \%$ $\mathrm{RDN}$ as FYM; $\mathrm{N}_{3}=50 \% \mathrm{RDF}+50 \% \mathrm{RDN}$ as FYM; vertical bars represent \pm standard error of the mean

(A)

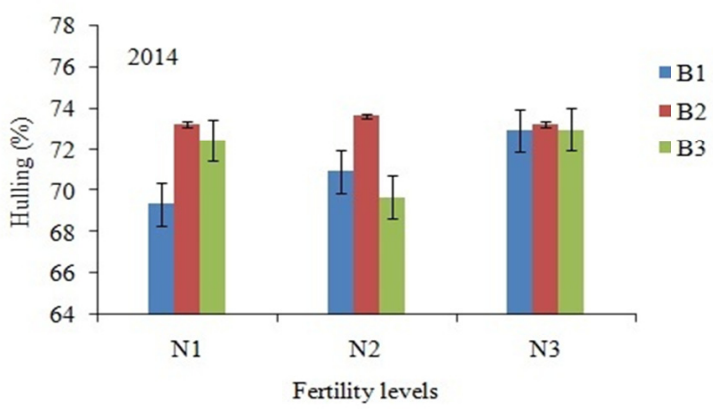

(B)

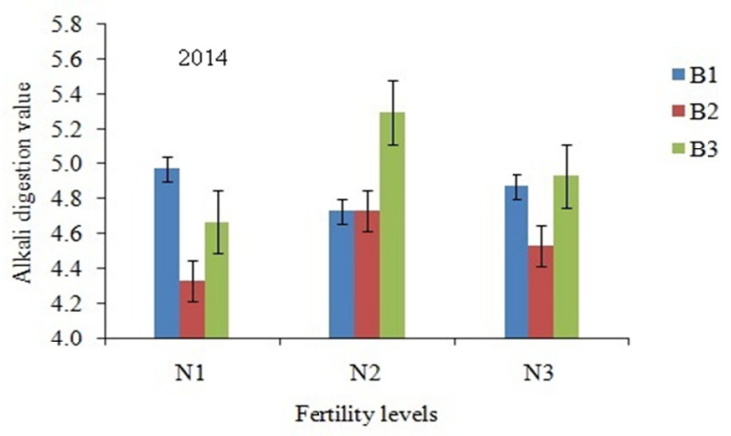

(C)

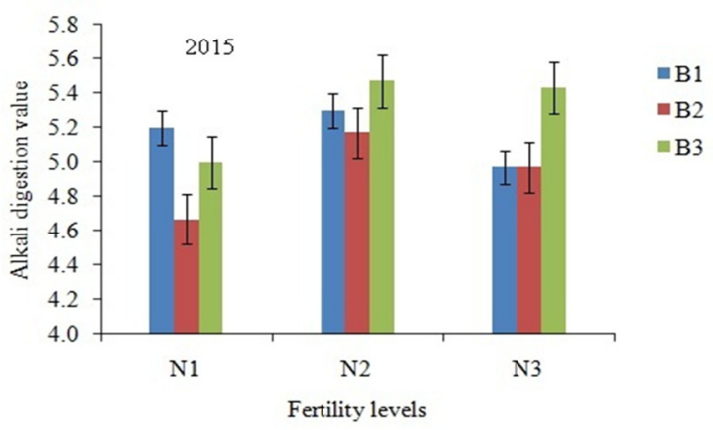

Figure 3. Interaction of fertility levels and bio-inoculants $(\mathrm{N} \times \mathrm{B})$ for $(\mathrm{A})$ hulling during $2014(\mathrm{p}<0.05)$; $(\mathrm{B})$ alkali digestion value during $2014(\mathrm{p}<0.05)$; (C) alkali digestion value during $2015(\mathrm{p}<0.05)$ of rice; $\mathrm{V}_{1}=$ HUBR 2-1; $\mathrm{V}_{2}=$ HUBR 10-9; $\mathrm{N}_{1}=100 \% \mathrm{RDF} ; \mathrm{N}_{2}=75 \% \mathrm{RDF}+25 \% \mathrm{RDN}$ as FYM; $\mathrm{N}_{3}=50 \% \mathrm{RDF}+50 \%$

$\mathrm{RDN}$ as FYM; vertical bars represent \pm standard error of the mean

\subsection{Kernel Length, Breadth Before and After Cooking and Elongation Ratio}

Kernel length and breadth before and after cooking were significantly greater with variety HUBR 10-9 (Table 2). Such differences in kernel and cooking characteristics among varieties are obvious (Gautam et al., 2008). HUBR 10-9 exhibited 5.6\% and 17.6\% greater elongation ratio than HUBR 2-1 during 2014 and 2015, respectively. More elongation without increase in girth is considered desirable cooking quality trait hence, higher elongation ratio is preferred (Hossain, Singh, \& Zaman, 2009). Fertility levels and bio-inoculants could not influence kernel length, breadth before and after cooking, and elongation ratio (Table 2). The interaction between genotype and environment governs the rice cooking quality traits (He et al., 1999). 
Table 2. Effect of varieties, fertility levels and bio-inoculants on kernel length before cooking (KLBC), kernel breadth before cooking (KBBC), kernel length after cooking (KLAC), kernel breadth after cooking (KBAC) and elongation ratio (ER) of rice

\begin{tabular}{|c|c|c|c|c|c|c|}
\hline & Treatments & $\mathrm{KLBC}(\mathrm{mm})$ & $\mathrm{KBBC}(\mathrm{mm})$ & $\operatorname{KLAC}(\mathrm{mm})$ & $\mathrm{KBAC}(\mathrm{mm})$ & ER \\
\hline \multirow[t]{11}{*}{2014} & Varieties & & & & & \\
\hline & $\mathrm{V}_{1}$ & $6.51 \pm 0.04^{\mathrm{b} *}$ & $1.85 \pm 0.01^{\mathrm{b}}$ & $10.33 \pm 0.16^{\mathrm{b}}$ & $2.82 \pm 0.02^{\mathrm{b}}$ & $1.59 \pm 0.02^{\mathrm{b}}$ \\
\hline & $\mathrm{V}_{2}$ & $6.73 \pm 0.04^{\mathrm{a}}$ & $1.91 \pm 0.01^{\mathrm{a}}$ & $11.30 \pm 0.16^{\mathrm{a}}$ & $2.92 \pm 0.02^{\mathrm{a}}$ & $1.68 \pm 0.02^{\mathrm{a}}$ \\
\hline & Fertility lev & & & & & \\
\hline & $\mathrm{N}_{1}$ & $6.61 \pm 0.05^{\mathrm{a}}$ & $1.88 \pm 0.02^{\mathrm{a}}$ & $10.76 \pm 0.19^{\mathrm{a}}$ & $2.88 \pm 0.03^{\mathrm{a}}$ & $1.63 \pm 0.03^{\mathrm{a}}$ \\
\hline & $\mathrm{N}_{2}$ & $6.67 \pm 0.05^{\mathrm{a}}$ & $1.89 \pm 0.02^{\mathrm{a}}$ & $10.97 \pm 0.19^{\mathrm{a}}$ & $2.91 \pm 0.03^{\mathrm{a}}$ & $1.65 \pm 0.03^{\mathrm{a}}$ \\
\hline & $\mathrm{N}_{3}$ & $6.58 \pm 0.05^{\mathrm{a}}$ & $1.87 \pm 0.02^{\mathrm{a}}$ & $10.70 \pm 0.19^{\mathrm{a}}$ & $2.84 \pm 0.03^{\mathrm{a}}$ & $1.63 \pm 0.03^{\mathrm{a}}$ \\
\hline & Bio-inocula & & & & & \\
\hline & $\mathrm{B}_{1}$ & $6.61 \pm 0.04^{\mathrm{a}}$ & $1.88 \pm 0.01^{\mathrm{a}}$ & $10.79 \pm 0.13^{\mathrm{a}}$ & $2.87 \pm 0.02^{\mathrm{a}}$ & $1.63 \pm 0.02^{\mathrm{a}}$ \\
\hline & $\mathrm{B}_{2}$ & $6.56 \pm 0.04^{\mathrm{a}}$ & $1.87 \pm 0.01^{\mathrm{a}}$ & $10.64 \pm 0.13^{\mathrm{a}}$ & $2.83 \pm 0.02^{\mathrm{a}}$ & $1.62 \pm 0.02^{\mathrm{a}}$ \\
\hline & $\mathrm{B}_{3}$ & $6.58 \pm 0.04^{\mathrm{a}}$ & $1.89 \pm 0.01^{\mathrm{a}}$ & $11.01 \pm 0.13^{\mathrm{a}}$ & $2.92 \pm 0.02^{\mathrm{a}}$ & $1.65 \pm 0.02^{\mathrm{a}}$ \\
\hline \multirow[t]{11}{*}{2015} & Varieties & & & & & \\
\hline & $\mathrm{V}_{1}$ & $6.97 \pm 0.04^{\mathrm{b}}$ & $1.90 \pm 0.01^{\mathrm{b}}$ & $11.09 \pm 0.07^{b}$ & $2.96 \pm 0.01^{\mathrm{b}}$ & $1.59 \pm 0.01^{\mathrm{b}}$ \\
\hline & $\mathrm{V}_{2}$ & $7.17 \pm 0.04^{\mathrm{a}}$ & $1.94 \pm 0.01^{\mathrm{a}}$ & $13.39 \pm 0.07^{\mathrm{a}}$ & $3.51 \pm 0.01^{\mathrm{a}}$ & $1.87 \pm 0.01^{\mathrm{a}}$ \\
\hline & Fertility lev & & & & & \\
\hline & $\mathrm{N}_{1}$ & $7.07 \pm 0.04^{\mathrm{a}}$ & $1.92 \pm 0.01^{\mathrm{a}}$ & $12.24 \pm 0.08^{\mathrm{a}}$ & $3.25 \pm 0.02^{\mathrm{a}}$ & $1.73 \pm 0.01^{\mathrm{a}}$ \\
\hline & $\mathrm{N}_{2}$ & $7.11 \pm 0.04^{\mathrm{a}}$ & $1.93 \pm 0.01^{\mathrm{a}}$ & $12.35 \pm 0.08^{\mathrm{a}}$ & $3.25 \pm 0.02^{\mathrm{a}}$ & $1.73 \pm 0.01^{\mathrm{a}}$ \\
\hline & $\mathrm{N}_{3}$ & $7.02 \pm 0.04^{\mathrm{a}}$ & $1.91 \pm 0.01^{\mathrm{a}}$ & $12.13 \pm 0.08^{\mathrm{a}}$ & $3.20 \pm 0.02^{\mathrm{a}}$ & $1.73 \pm 0.01^{\mathrm{a}}$ \\
\hline & Bio-inocula & & & & & \\
\hline & $\mathrm{B}_{1}$ & $7.07 \pm 0.03^{\mathrm{a}}$ & $1.92 \pm 0.01^{\mathrm{a}}$ & $12.24 \pm 0.07^{\mathrm{a}}$ & $3.24 \pm 0.03^{\mathrm{a}}$ & $1.73 \pm 0.01^{\mathrm{a}}$ \\
\hline & $\mathrm{B}_{2}$ & $7.03 \pm 0.03^{\mathrm{a}}$ & $1.91 \pm 0.01^{\mathrm{a}}$ & $12.14 \pm 0.07^{\mathrm{a}}$ & $3.21 \pm 0.03^{\mathrm{a}}$ & $1.73 \pm 0.01^{\mathrm{a}}$ \\
\hline & $\mathrm{B}_{3}$ & $7.11 \pm 0.03^{\mathrm{a}}$ & $1.93 \pm 0.01^{\mathrm{a}}$ & $12.34 \pm 0.07^{\mathrm{a}}$ & $3.25 \pm 0.03^{\mathrm{a}}$ & $1.73 \pm 0.01^{\mathrm{a}}$ \\
\hline
\end{tabular}

Note. $*$ Mean values \pm SE. Mean values with different superscript letters denote significant difference $(\mathrm{p}<0.05)$. $\mathrm{V}_{1}=$ HUBR 2-1; $\mathrm{V}_{2}=$ HUBR $10-9 ; \mathrm{N}_{1}=100 \% \mathrm{RDF} ; \mathrm{N}_{2}=75 \% \mathrm{RDF}+25 \% \mathrm{RDN}$ as FYM; $\mathrm{N}_{3}=50 \% \mathrm{RDF}+$ $50 \% \mathrm{RDN}$ as FYM; $\mathrm{B}_{1}=\mathrm{BGA} ; \mathrm{B}_{2}=$ Azospirillum $; \mathrm{B}_{3}=\mathrm{BGA}+$ Azospirillum.

\subsection{Protein Content and Yield}

In spite of advanced approaches used for enhancement in the rice grain nutritional traits, so far little success achieved due to complex inheritance. Protein content is influenced largely due to effect of environment (Mahender, Anandan, Pradhan, \& Pandit, 2016). Grain protein content and yield of basmati rice varieties differed significantly due to varied fertility levels and bio-inoculants (Table 3). HUBR 2-1 showed higher protein content over HUBR 10-9 because of more N content in grain. Higher protein yield noted by HUBR $10-9$ that is $12.2 \%$ and $11.9 \%$ over HUBR $2-1$ during first and second year, respectively due to better grain yield. Similar differences in protein content of cultivars were also noted (Ghosh et al., 2004; Chandel et al., 2010; Neupane, Singh, \& Sravan, 2017). Fertility levels followed the order $N_{2}>N_{1}>N_{3}$ for protein content and yield during both years (Table 3). Higher protein content and yield with $\mathrm{N}_{2}$ might be due to continuous $\mathrm{N}$ supply and its efficient translocation to grain; greater nitrogen content in grain customized the proportion of grain constituents (S. K. Yadav, Y. Singh, Kumar, M. K. Yadav, \& K. Singh, 2013). Combined application $\left(\mathrm{B}_{3}\right)$ improved protein content and yield than $\mathrm{B}_{1}$ and $\mathrm{B}_{2}$ in both the years. Synergistic effect of bio-inoculants caused consistent release of nitrogen through decomposition of the cells (Nayak et al., 2004). Similar response of nitrogen fertilizer and bio-fertilizers for protein content was also noted (Hemalatha, Thirumurugan, \& Balasubramanian, 1999; Hargilas \& Sharma, 2015). 
Table 3. Effect of varieties, fertility levels and bio-inoculants on grain protein content (GPC), protein yield (PY), amylose content $(\mathrm{AC})$ and alkali digestion value (ADV) of rice

\begin{tabular}{|c|c|c|c|c|c|}
\hline & Treatments & GPC (\%) & $\mathrm{PY}\left(\mathrm{kg} \mathrm{ha}^{-1}\right)$ & $\mathrm{AC}(\%)$ & $\mathrm{ADV}$ \\
\hline \multirow[t]{11}{*}{2014} & Varieties & & & & \\
\hline & $\mathrm{V}_{1}$ & $7.98 \pm 0.11^{\mathrm{a} *}$ & $382.66 \pm 8.20^{\mathrm{b}}$ & $20.60 \pm 0.13^{\mathrm{b}}$ & $4.45 \pm 0.05^{\mathrm{b}}$ \\
\hline & $\mathrm{V}_{2}$ & $7.50 \pm 0.11^{\mathrm{b}}$ & $429.31 \pm 8.20^{\mathrm{a}}$ & $22.02 \pm 0.13^{\mathrm{a}}$ & $5.12 \pm 0.05^{\mathrm{a}}$ \\
\hline & Fertility levels & & & & \\
\hline & $\mathrm{N}_{1}$ & $7.79 \pm 0.14^{\mathrm{ab}}$ & $406.81 \pm 10.05^{\mathrm{b}}$ & $20.93 \pm 0.15^{\mathrm{b}}$ & $4.66 \pm 0.06^{\mathrm{b}}$ \\
\hline & $\mathrm{N}_{2}$ & $7.99 \pm 0.14^{\mathrm{a}}$ & $446.48 \pm 10.05^{\mathrm{a}}$ & $21.65 \pm 0.15^{\mathrm{a}}$ & $4.92 \pm 0.06^{\mathrm{a}}$ \\
\hline & $\mathrm{N}_{3}$ & $7.44 \pm 0.14^{\mathrm{b}}$ & $364.66 \pm 10.05^{\mathrm{c}}$ & $21.36 \pm 0.15^{\mathrm{ab}}$ & $4.78 \pm 0.06^{\mathrm{ab}}$ \\
\hline & Bio-inoculants & & & & \\
\hline & $\mathrm{B}_{1}$ & $7.62 \pm 0.12^{\mathrm{b}}$ & $396.83 \pm 9.34^{\mathrm{b}}$ & $21.34 \pm 0.09^{\mathrm{ab}}$ & $4.86 \pm 0.07^{\mathrm{a}}$ \\
\hline & $\mathrm{B}_{2}$ & $7.57 \pm 0.12^{\mathrm{b}}$ & $385.55 \pm 9.34^{\mathrm{b}}$ & $21.10 \pm 0.09^{\mathrm{b}}$ & $4.53 \pm 0.07^{b}$ \\
\hline & $\mathrm{B}_{3}$ & $8.03 \pm 0.12^{\mathrm{a}}$ & $435.58 \pm 9.34^{\mathrm{a}}$ & $21.49 \pm 0.09^{\mathrm{a}}$ & $4.97 \pm 0.07^{\mathrm{a}}$ \\
\hline \multirow[t]{11}{*}{2015} & Varieties & & & & \\
\hline & $\mathrm{V}_{1}$ & $8.02 \pm 0.10^{\mathrm{a}}$ & $406.23 \pm 11.06^{\mathrm{b}}$ & $20.71 \pm 0.15^{\mathrm{b}}$ & $4.77 \pm 0.06^{\mathrm{b}}$ \\
\hline & $\mathrm{V}_{2}$ & $7.63 \pm 0.10^{\mathrm{b}}$ & $454.47 \pm 11.06^{\mathrm{a}}$ & $22.81 \pm 0.15^{\mathrm{a}}$ & $5.49 \pm 0.06^{\mathrm{a}}$ \\
\hline & Fertility levels & & & & \\
\hline & $\mathrm{N}_{1}$ & $7.87 \pm 0.12^{\mathrm{ab}}$ & $431.32 \pm 13.55^{\mathrm{b}}$ & $21.37 \pm 0.18^{\mathrm{b}}$ & $4.96 \pm 0.08^{\mathrm{b}}$ \\
\hline & $\mathrm{N}_{2}$ & $8.05 \pm 0.12^{\mathrm{a}}$ & $476.07 \pm 13.55^{\mathrm{a}}$ & $22.12 \pm 0.18^{\mathrm{a}}$ & $5.31 \pm 0.08^{\mathrm{a}}$ \\
\hline & $\mathrm{N}_{3}$ & $7.55 \pm 0.12^{\mathrm{b}}$ & $383.65 \pm 13.55^{\mathrm{c}}$ & $21.79 \pm 0.18^{\mathrm{ab}}$ & $5.12 \pm 0.08^{\mathrm{ab}}$ \\
\hline & Bio-inoculants & & & & \\
\hline & $\mathrm{B}_{1}$ & $7.72 \pm 0.12^{\mathrm{b}}$ & $424.29 \pm 12.34^{\mathrm{b}}$ & $21.76 \pm 0.11^{\mathrm{ab}}$ & $5.16 \pm 0.06^{\mathrm{a}}$ \\
\hline & $\mathrm{B}_{2}$ & $7.68 \pm 0.12^{\mathrm{b}}$ & $405.03 \pm 12.34^{b}$ & $21.50 \pm 0.11^{\mathrm{b}}$ & $4.93 \pm 0.06^{\mathrm{b}}$ \\
\hline & $\mathrm{B}_{3}$ & $8.08 \pm 0.12^{\mathrm{a}}$ & $461.73 \pm 12.34^{\mathrm{a}}$ & $22.02 \pm 0.11^{\mathrm{a}}$ & $5.30 \pm 0.06^{\mathrm{a}}$ \\
\hline
\end{tabular}

Note. *Mean values \pm SE. Mean values with different superscript letters denote significant difference $(\mathrm{p}<0.05)$. $\mathrm{V}_{1}=$ HUBR 2-1; $\mathrm{V}_{2}=$ HUBR 10-9; $\mathrm{N}_{1}=100 \%$ RDF; $\mathrm{N}_{2}=75 \% \mathrm{RDF}+25 \% \mathrm{RDN}$ as FYM; $\mathrm{N}_{3}=50 \% \mathrm{RDF}+$ $50 \% \mathrm{RDN}$ as FYM; $\mathrm{B}_{1}=\mathrm{BGA} ; \mathrm{B}_{2}=$ Azospirillum; $\mathrm{B}_{3}=\mathrm{BGA}+$ Azospirillum.

\subsection{Amylose Content and Alkali Digestion Value}

Amylose content is an important index to judge rice cooking and processing behaviour and determines the cohesiveness, tenderness, stickiness, colour and gloss of the boiled rice (Denyer, Johnson, Zeeman, \& Smith, 2001). Varieties varied in amylose content and alkali digestion value because of fertility levels and bio-inoculants (Table 3). HUBR 10-9 showed higher amylose content (6.9\% and 10.1\%) and alkali digestion values (5.12 and 5.49) than HUBR 2-1 during first and second year, respectively. Values indicate medium disintegration of kernel and intermediate alkali digestion values which are highly desirable for grain quality (Bhonsle, 2010; Singh, Singh, Nandan, \& Rao, 2012). Integration of inorganic with organic nutrient source $\left(\mathrm{N}_{2}\right)$ caused highest amylose and alkali digestion value (Table 3). Enhanced quality parameters with combined use of organic and inorganic were also reported (Quyen \& Sharma, 2003). Varied response to fertility management regarding quality traits of two genotypes was obvious. Apart from crop management, the nitrogen management had significant impact on grain quality parameters ( $\mathrm{Gu}$ et al., 2015). Use of two bio-inoculants together $\left(\mathrm{B}_{3}\right)$ produced highest amylose content and alkali digestion value. Secretion of growth promoting substances in addition to biological nitrogen fixation might have helped in improved quality (Hemalatha et al., 1999; Hargilas \& Sharma, 2015). The interaction between fertility levels and bio-inoculants found significant during both years for alkali digestion value (Figures 3B and 3C). Combined effect of inorganic, organic and bio-inoculants caused continuous supply of nutrients throughout the growth period resulted better quality. Similar findings reported by Davari and Sharma (2010) and Hargilas and Sharma (2015).

\section{Conclusion}

The two genotypes responded differently to varied combinations of fertility and the bio-inoculants. Basmati genotype HUBR 10-9 showed considerably higher yield potential and possessed superior quality characters than HUBR 2-1. Results suggest that the genotypic factors govern the grain quality traits. Application of recommended nutrients in integrated approach (75\% RDF plus 25\% FYM) enhanced grain yield and the quality traits. Combined application of bio-inoculants (BGA plus Azospirillum) showed synergistic effect and improved the grain yield and quality over alone application of BGA or Azospirillum. Significant interactions noticed to 
influence the grain quality traits. Study suggest, basmati rice HUBR 10-9 be grown with integrated nutrient management that is $75 \%$ RDF plus 25\% RDN as FYM along with BGA plus Azospirillum to exploit the potential yield and quality under conditions of eastern Uttar Pradesh. Evaluation of the traditional basmati cultivars with above genotypes required for comparison of yield potential and quality traits. Further, studies under diverse conditions required to analyze the performance of these genotypes in traditional and non-traditional potential areas.

\section{References}

Adhikari, N. P., Mishra, B. N., \& Mishra, P. K. (2005). Effect of integrated nitrogen management on quality of aromatic rice. Annals of Agricultural Research, 26, 231-234.

Ahmed, N., Tetlow, I. J., Nawaz, S., Iqbal, A., Mubin, M., Rehman, M. S. N., .. Maekawa, M. (2015). Effect of high temperature on grain filling period, yield, amylose content and activity of starch biosynthesis enzymes in endosperm of basmati rice. Journal of the Science of Food and Agriculture, 95, 2237-2243. https://doi.org/10.1002/jsfa.6941

AOAC (Association of Official Analytical Chemists). (1990). Official Methods of Chemical Analysis (15th ed.). AOAC, Arlington, VA.

Bhonsle, S. J. (2010). Grain quality evaluation and organoleptic analysis of aromatic rice varieties of Goa, India. Journal of Agricultural Science, 2, 99-107. https://doi.org/10.5539/jas.v2n3p99

Binodh, A. K., Kalaiyarasi, R., Thiyagarajan, K., \& Manonmani, S. (2006). Physico-chemical and cooking quality characteristics of promising varieties and hybrids in rice (Oryza sativa L.). Indian Journal of Genetics and Plant Breeding, 66, 107-112.

Chandel, G., Banerjee, S., See, S., Meena, R., Sharma, D. J., \& Verulkar, S. B. (2010). Effects of different nitrogen fertilizer levels and native soil properties on rice grain Fe, $\mathrm{Zn}$ and protein contents. Rice Science, 17, 213-227. https://doi.org/10.1016/S1672-6308(09)60020-2

Davari, M. R., \& Sharma, S. N. (2010). Effect of different combinations of organic materials and biofertilizers on productivity, grain quality and economics in organic farming of basmati rice (Oryza sativa). Indian Journal of Agronomy, 55, 290-294.

Dela Cruz, N., \& Khush, G. S. (2000). Rice grain quality evaluation procedures. In R. K. Singh, U. S. Singh, \& G. S. Khush (Eds.), Aromatic rices (pp. 15-28). Oxford and IBH publishing Co. Pvt. Ltd., New Delhi.

Dela Cruz, N., Kumar, I., Kaushik, R. P., \& Khush, G. S. (1989). Effect of temperature during grain development on the performance and stability of cooking quality components of rice. Japanese Journal of Breeding, 39, 299-306. https://doi.org/10.1270/jsbbs1951.39.299

Denyer, K., Johnson, P., Zeeman, S., \& Smith, A. M. (2001). The control of amylose synthesis. Journal of Plant Physiology, 158, 479-487. https://doi.org/10.1078/0176-1617-00360

Dixit, K. G., \& Gupta, B. R. (2000). Effect of farmyard manure, chemical and biofertilizers on yield and quality of rice (Oryza sativa) and soil properties. Journal of the Indian Society of Soil Science, 48, 773-780.

Fan, J., Siebenmorgen, T. J., \& Yang, W. (2000). A study of head rice yield reduction of long-and medium-grain varieties in relation to various harvest and drying conditions. Transactions of the ASAE. American Society of Agricultural Engineers, 43, 1709-1714. https://doi.org/10.13031/2013.3072

Gautam, A. K., Kumar, D., Shivay, Y. S., \& Mishra, B. N. (2008). Influence of nitrogen levels and plant spacing on growth, productivity and quality of two inbred varieties and a hybrid of aromatic rice. Archives of Agronomy and Soil Science, 54, 515-532. https://doi.org/10.1080/03650340802283470

Ghosh, M., Mandal, B. K., Mandal, B. B., Lodh, S. B., \& Dash, A. K. (2004). The effect of planting date and nitrogen management on yield and quality of aromatic rice (Oryza sativa). The Journal of Agricultural Science, 142, 183-191. https://doi.org/10.1017/S002185960400423X

Gomez, K. A., \& Gomez, A. A. (1984). Statistical procedures for agricultural research (2nd ed.). John Willey and Sons, New York.

Gu, J., Chen, J., Chen, L., Wang, Z., Zhang, H., \& Yang, J. (2015). Grain quality changes and responses to nitrogen fertilizer of japonica rice cultivars released in the Yangtze River Basin from the 1950s to 2000s. The Crop Journal, 3, 285-297. https://doi.org/10.1016/j.cj.2015.03.007 
Hargilas, \& Sharma, S. N. (2015). Effect of different combinations of organic manures and biofertlizers on growth, yield, grain quality and economics in organic farming of scented rice. Journal of Plant Development Sciences, 7, 381-388.

He, P., Li, S. G., Qian, Q., Ma, Y. Q., Li, J. Z., Wang, W. M., ... Zhu, L. H. (1999). Genetic analysis of rice grain quality. Theoretical and Applied Genetics, 98, 502-508. https://doi.org/10.1007/s001220051098

Heinemann, R. J. B., Fagundes, P. L., Pinto, E. A., Penteado, M. V. C., \& Lanfer-Marquez, U. M. (2005). Comparative study of nutrient composition of commercial brown, parboiled and milled rice from Brazil. Journal of Food Composition and Analysis, 18, 287-296. https://doi.org/10.1016/j.jfca.2004.07.005

Hemalatha, M., Thirumurugan, V., \& Balasubramanian, R. (1999). Influence of organic, biofertilizer, and inorganic forms of nitrogen on rice quality. International Rice Research Notes, 24, 33.

Hossain, M. S., Singh, A. K., \& Zaman, F. U. (2009). Cooking and eating characteristics of some newly identified inter sub-specific (indica/japonica) rice hybrids. Science Asia, 35, 320-325. https://doi.org/ 10.2306/scienceasia1513-1874.2009.35.320

Jackson, M. L. (1973). Soil Chemical Analysis. Prentice Hall of India Pvt. Ltd., New Delhi.

Juliano, B. O., \& Perez, C. M. (1984). Results of a collaborative test on the measurement of grain elongation of milled rice during cooking. Journal of Cereal Science, 2, 281-292. https://doi.org/10.1016/S0733-5210 (84)80016-8

Juliano, B. O. (1971). A simplified assay for milled rice amylose. Cereal Science Today, 16, 334-338.

Juliano, B. O. (1972). Physico-chemical properties of starch and protein in relation to grain quality and nutritional value of rice. IRRI Rice Breeding (pp. 389-404). Los Banos, Philippines.

Khush, G. S., Paule, C. M., \& Dela Cruz, N. M. (1979). Rice grain quality evaluation and improvement at IRRI. Proceedings of workshop in Chemical Aspects of Rice Grain Quality (pp. 22-31). International Rice Research Institute, Los Banos, Laguna, Philippines.

Mahender, A., Anandan, A., Pradhan, S. K., \& Pandit, E. (2016). Rice grain nutritional traits and their enhancement using relevant genes and QTLs through advanced approaches. Springer Plus, 5, 2086. https://doi.org/10.1186/s40064-016-3744-6

Murthy, M. G., \& Ladha, J. K. (1988). Influence of Azospirillum inoculation on mineral uptake and growth of rice under hydroponic conditions. Plant and Soil, 108, 281-285. https://doi.org/10.1007\%2FBF02375660

Nanda, G., Sravan, U. S., Singh, A., \& Singh, S. P. (2016). Effect of NPK levels and bio-organics on growth, yield and economics of basmati rice (Oryza sativa L.) cv HUBR 10-9. Environment and Ecology, 34, 1530-1534.

Nayak, S., Prasanna, R., Pabby, A., Dominic, T. K., \& Singh, P. K. (2004). Effect of urea, blue green algae and Azolla on nitrogen fixation and chlorophyll accumulation in soil under rice. Biology and Fertility of Soils, 40, 67-72. https://doi.org/ 10.1007/s00374-004-0738-2

Neupane, M. P., Singh, S. P., \& Sravan, U. S. (2017). Response of baby corn genotypes to soil and foliar nitrogen application schedule. Archives of Agronomy and Soil Science, 63, 1912-1926. https://doi.org/10.1080/ 03650340.2017 .1311011

Pandey, N., Sarawgi, A. K., Rastogi, N. K., \& Tripathi, R. S. (1999). Effect of farmyard manure and chemical N fertilizer on grain yield and quality of scented rice (Oryza sativa) varieties. Indian Journal of Agricultural Sciences, 69, 621-623.

Perez, C. M., Juliano, B. O., Liboon, S. P., Alcantara, J. M., \& Cassman, K. G. (1996). Effects of late nitrogen fertilizer application on head rice yield, protein content and grain quality of rice. Cereal Chemistry, 73, 556-560.

Prakash, Y. S., Bhadoria, P. B. S., \& Rakshit, T. (2002). Relative efficacy of organic manures in improving milling and cooking quality of rice. International Rice Research Notes, 27, 43-44.

Quyen, N. V., \& Sharma, S. N. (2003). Relative effect of organic and conventional farming on growth, yield and grain quality of scented rice and soil fertility. Archives of Agronomy and Soil Science, 49, 623-629. https://doi.org/10.1080/03650340310001612979 
Saha, S., Pandey, A. K., Gopinath, K. A., Bhattacharaya, R., Kundu, S., \& Gupta, H. S. (2007). Nutritional quality of organic rice grown on organic composts. Agronomy for Sustainable Development, 27, 223-229. https://doi.org/10.1051/agro:2007002

Siebenmorgen, T. J., Jia, C., Qin, G., \& Schluterman, D. (2006). Evaluation of selected rice laboratory shelling equipment. Applied Engineering in Agriculture, 22, 427-431. https://doi.org/10.13031/2013.20445

Singh, A. K., Singh, P. K., Nandan, R., \& Rao, M. (2012). Grain quality and cooking properties of rice germplasm. Annals of Plant and Soil Research, 14, 52-57.

Singh, D. K., Pandey, P. C., Nanda, G., \& Gupta, S. (2018). Long-term effects of inorganic fertilizer and farmyard manure application on productivity, sustainability and profitability of rice-wheat system in Mollisols. Archives of Agronomy and Soil Science, 65, 139-151. https://doi.org/10.1080/03650340. 2018.1491032

Singh, F., Kumar, R., \& Pal, S. (2008). Integrated nutrient management in rice-wheat cropping system for sustainable productivity. Journal of the Indian Society of Soil Science, 56, 205-208.

Singh, R. P., Jaiswal, H. K., \& Madhavilatha, L. (2006). HUBR 2-1 (Malviya Basmati Dhan 1), a new, high-yielding basmati rice variety for cultivation in eastern India. International Rice Research Notes, 31(2), 33-34.

Srivastava, P., Srivastava, P. C., Singh, U. S., \& Shrivastava, M. (2009). Effect of integrated and balanced nutrient application on soil fertility, yield and quality of basmati rice. Archives of Agronomy and Soil Science, 55, 265-284. https://doi.org/10.1080/03650340802477734

Surekha, K., Rao, K. V., Shobha Rani, N., Latha, P. C., \& Kumar, R. M. (2013). Evaluation of organic and conventional rice production systems for their productivity, profitability, grain quality and soil health. Agrotechnology, S11, 1-6. https://doi.org/10.4172/2168-9881.S11-006

Tang, H., Xiao, X., Tang, W., Li, C., Wang, K., Li, W., ... Pan, X. (2018). Long-term effects of NPK fertilizers and organic manures on soil organic carbon and carbon management index under a double-cropping rice system in Southern China. Communications in Soil Science and Plant Analysis, 49, 1976-1989. https://doi.org/10.1080/00103624.2018.1492600

Yadav, S. K., Singh, Y., Kumar, R. P., Yadav, M. K., \& Singh, K. (2013). Effect of organic nitrogen sources on yield quality and nutrient uptake of rice (Oryza sativa) under different cropping system. Vegetos, 26, 58-66. https://doi.org/10.5958/j.2229-4473.26.1.009

Yaduvanshi, N. P. S. (2003). Substitution of inorganic fertilizers by organic manures and the effect on soil fertility in a rice-wheat rotation on reclaimed sodic soil in India. The Journal of Agricultural Science, 140, 161-168. https://doi.org/10.1017/S0021859603002934

Zhou, L., Liang, S., Ponce, K., Marundon, S., Ye, G., \& Zhao, X. (2015). Factors affecting head rice yield and chalkiness in indica rice. Field Crops Research, 172, 1-10. https://doi.org/10.1016/j.fcr.2014.12.004

\section{Copyrights}

Copyright for this article is retained by the author(s), with first publication rights granted to the journal.

This is an open-access article distributed under the terms and conditions of the Creative Commons Attribution license (http://creativecommons.org/licenses/by/4.0/). 